\title{
Iron regulates the expression of ferroportin 1 in the cultured hFOB 1.19 osteoblast cell line
}

\author{
GUO-YANG ZHAO $^{1}$, DONG-HUA DI ${ }^{1}$, BO WANG ${ }^{1}$, PENG ZHANG $^{2}$ and YOU-JIA XU ${ }^{2}$ \\ ${ }^{1}$ Department of Orthopedics, The Affiliated Hospital of Jiangsu University, Zhenjiang, Jiangsu 212000; \\ ${ }^{2}$ Department of Orthopedics, The Second Affiliated Hospital of Soochow University, \\ Suzhou, Jiangsu 215004, P.R. China
}

Received February 1, 2014; Accepted June 11, 2014

DOI: $10.3892 / \mathrm{etm} .2014 .1823$

\begin{abstract}
Iron metabolism is tightly regulated in osteoblasts, and ferroportin 1 (FPN1) is the only identified iron exporter in mammals to date. In the present study, the regulation of FNP1 in human osteoblasts was investigated following various iron treatments. The human osteoblast cell line hFOB 1.19 was treated with ferric ammonium citrate (FAC) or desferrioxamine (DFO) of various concentrations. The intracellular iron ion levels were measured using a confocal laser scanning microscope. In addition, the mRNA and protein expression levels of FPN1 were detected by quantitative polymerase chain reaction, western blot analysis and immunofluorescence. The results demonstrated that increasing iron concentrations via FAC treatment increased the expression of FPN1. By contrast, decreasing the iron concentration by DFO treatment decreased FNP1 expression levels. In addition to demonstrating that the FNP1 expression changed according to the iron concentration, the observations indicated that changes in FPN1 expression may contribute to the maintenance of the intracellular iron balance in osteoblasts.
\end{abstract}

\section{Introduction}

Iron is one of the most important trace elements found in the human body. As with all cells, bone cells require iron for numerous aspects of their physiology. A number of studies have indicated that bone metabolism is closely associated with iron metabolism. In particular, iron overload and iron deficiency may lead to osteopenia or even osteoporosis (1-3). Iron chelation therapy has been demonstrated to improve osteoporosis in ovariectomized rats $(4,5)$. In addition, hepcidin (a peptide hormone that decreases iron levels in the body) has also been investigated for the treatment of osteoporosis in peri- and

Correspondence to: Professor You-Jia Xu, Department of Orthopedics, The Second Affiliated Hospital of Soochow University, 1055 Sanxiang Road, Suzhou, Jiangsu 215004, P.R. China

E-mail:xuyoujia@medmail.com.cn

Key words: iron metabolism, ferroportin 1, osteoblast post-menopausal females (6). These studies indicate that iron plays an important role in bone metabolism. Therefore, research into the mechanisms underlying the iron balance in bone cells is crucial to improve the understanding of the pathogenesis and treatment of iron-associated bone disease.

In vitro studies have previously revealed that iron excess inhibits osteoblastic metabolism due to the damage caused by oxidative stress (7-9), while iron deficiency can inhibit osteoblastogenesis due to the decreased activity of ribonucleotide reductase $(10,11)$. Ferroportin 1 (FPN1), which contributes to iron release from cells and the maintenance of iron homeostasis, is currently the only iron exporter to be identified in mammals (12-14). The exporter is highly expressed in macrophages, enterocytes and hepatocytes $(15,16)$. A recent study demonstrated that FPN1 is also expressed in human osteoblasts (17). The expression of FPN1 in macrophages (18-20), enterocytes $(21,22)$, hepatocytes $(23)$ and cardiocytes $(24)$ has been reported to be regulated by iron concentration; however, the association between FPN1 and iron ion levels in osteoblasts is yet to be fully elucidated. In the present study, the human osteoblast cell line hFOB 1.19 was treated with ferric ammonium citrate (FAC) or desferrioxamine (DFO) of various concentrations. The intracellular levels of iron ions were measured using confocal laser scanning microscopy (CLSM). In addition, the mRNA and protein expression levels of FPN1 were detected by quantitative polymerase chain reaction (qPCR), western blot analysis and immunofluorescence. The aim of the present study was to provide further information to improve the understanding of the role that FPN1 plays in osteoblastic iron metabolism.

\section{Materials and methods}

Cell cultures and treatments. The hFOB 1.19 cell line (Shanghai Institute of Biochemistry and Cell Biology, Shanghai, China) was maintained in Dulbecco's modified Eagle's medium- $\mathrm{F}_{12}$, supplemented with $10 \%$ fetal bovine serum and 3\% G418 disulfate solution, in a humidified atmosphere of $5 \% \mathrm{CO}_{2}$ in air at $34^{\circ} \mathrm{C}$. The medium was replenished every 2-3 days. After reaching 70-80\% confluence, the cells were passaged by treatment with $0.05 \%$ trypsin. For CLSM, qPCR and western blot analysis, FAC (Sinopharm Chemical Reagent Co. Ltd., Shanghai, China) and DFO (Novartis Pharma Schweiz AG, 
Rotkreuz, Switzerland) were added to the medium at final concentrations of 50,100 and $200 \mu \mathrm{mol} / 1$ for FAC as treatment in the iron excess group and 5, 10 and $20 \mu \mathrm{mol} / 1$ for DFO as treatment in the iron deficiency group. For immunofluorescence FPN1 analysis, $50 \mu \mathrm{mol} / 1 \mathrm{FAC}$ and $10 \mu \mathrm{mo} / \mathrm{l} \mathrm{DFO}$ were added for the iron excess and iron deficiency groups, respectively. In the control, the same amount of medium without FAC or DFO was used. Cells were incubated with FAC and DFO for $20 \mathrm{~h}$.

Confocal microcopy measurements. The hFOB 1.19 cells were seeded on coverslips for the analysis of iron ions by fluorescence quenching. Briefly, following treatment with FAC and DFO for $20 \mathrm{~h}$, the hFOB 1.19 cells were washed twice with phosphate-buffered saline (PBS) and incubated with Phen Green FL (Molecular Probes, Eugene, OR, USA) away from light at $34^{\circ} \mathrm{C}$ in a humidified atmosphere containing $5 \% \mathrm{CO}_{2}$ for $30 \mathrm{~min}$. Next, the cells were washed twice with PBS to remove the unbound fluorescent indicator, and then incubated with the culture medium for an additional $15 \mathrm{~min}$. CLSM model TCS-SP2 (Leica, Wetzlar, Germany) was used to measure the green fluorescence of Phen Green FL when excited at $488 \mathrm{~nm}$ and emitted at $521 \mathrm{~nm}$.

qPCR analysis. Total RNA was extracted from the hFOB 1.19 cells following treatment using TRIzol reagent (Invitrogen Life Technologies, Carlsbad, CA, USA) and single-stranded cDNA was synthesized using a reverse transcription kit purchased from Promega Corporation (Madison, WI, USA), according to the manufacturer's instructions. qPCR was performed using a real-time PCR system (Applied Biosystems Step One; Thermo Fisher Scientific, Waltham, MA, USA). Amplification reactions were conducted in a $20-\mu 1$ volume using SYBR-Green I dye under the following amplification conditions: 30 cycles of $94^{\circ} \mathrm{C}$ for $30 \mathrm{sec}, 50^{\circ} \mathrm{C}$ for $30 \mathrm{sec}$ and $72^{\circ} \mathrm{C}$ for $30 \mathrm{sec}$. Primers were designed to specifically amplify $176 \mathrm{bp}$ of human FPN1 cDNA (forward, 5'-CTACTTGGGGAGATCGGATGT-3' and reverse, 5'-CTGGGCCACTTTAAGTCTAGC-3'); and 306 bp of human $\beta$-actin cDNA (forward, 5'-TCCTGTGGCATC CACGAAACT-3' and reverse, 5'-GAAGCATTTGCGGTG GACGAT-3'). The mRNA/cDNA abundance of each gene was calculated relative to the expression of the housekeeping gene, $\beta$-actin. Relative quantification was calculated using the $2^{-\triangle \Delta C T}$ method and analysis was performed using Step One ${ }^{\mathrm{TM}}$ Software V 2.1 (Thermo Fisher Scientific).

Western blot analysis. Total protein was extracted from the hFOB 1.19 cells following treatment using radioimmunoprecipitation assay buffer, and separated on 6\% SDS gel prior to transfer onto polyvinylidene difluoride membranes. The membranes were blocked in $5 \%(\mathrm{~m} / \mathrm{v})$ milk dissolved in Tris-buffered saline with $0.05 \%(\mathrm{w} / \mathrm{v})$ Tween-20 (TBS-T) and incubated overnight at $4^{\circ} \mathrm{C}$ with rabbit anti-ferroportin, (1:200) or anti- $\beta$-actin (1:500; Abcam, Cambridge, MA, USA) primary antibodies. Following washing three times with TBS-T at room temperature, the membranes were incubated for $1 \mathrm{~h}$ with goat peroxidase-labeled anti-rabbit immunoglobulin (1:500), and visualized with enhanced chemiluminescence (Amersham Biosciences Corporation, Piscataway, NJ, USA). The images were analyzed with ImageJ software (National Institutes of Health, Bethesda, MD, USA).
Immunofluorescence analysis. Cells were seeded on glass coverslips for immunofluorescence analysis. Following treatment, the cells were fixed with $4 \%$ paraformaldehyde for $15 \mathrm{~min}$ and washed twice with PBS. The cells were then incubated in a blocking solution (5\% bovine serum albumin) for $30 \mathrm{~min}$ at room temperature, followed by incubation with primary antibodies (rabbit anti-ferroportin 1; 1:50; Abcam) in a humid chamber at $4^{\circ} \mathrm{C}$ overnight. Following washing three times with PBS, the cells were incubated with a fluorescein isothiocyanate-conjugated goat anti-rabbit antibody $(1: 1,000$; Jackson ImmunoResearch Laboratories, Inc., West Grove, PA, USA) at room temperature for $30 \mathrm{~min}$. Coverslips were then washed three times with PBS, mounted and observed using a fluorescent microscope (Axio Observer A1; Carl Zeiss AG, Oberkochen, Germany).

Statistical analysis. Data are expressed as the mean \pm standard deviation, and were analyzed with one-way analysis of variance with post-hoc analysis using SPSS version 15.01 for Windows (SPSS, Inc., Chicago, IL, USA). P<0.05 was considered to indicate a statistically significant difference.

\section{Results}

Intracellular fluorescence quenching by iron following treatment with FAC and DFO. The hFOB 1.19 cells in culture exhibited typical spindle and polygon shapes. Following exposure to various concentrations of FAC and DFO for $20 \mathrm{~h}$, a correlation between the fluorescence intensity in the hFOB 1.19 cells and the intracellular iron concentration was observed; the fluorescence intensity significantly weakened with increasing FAC concentrations, but was enhanced with increasing DFO concentrations $(\mathrm{P}<0.05$ for all comparisons; Fig. 1). These observations indicate that FAC effectually increased the intracellular iron concentration, while DFO effectually decreased the intracellular iron levels.

$m R N A$ and protein expression levels of FPN1 following treatment with FAC and DFO. qPCR revealed that the mRNA expression levels of FPN1 in osteoblasts increased with increasing concentrations of FAC in a concentration-dependent manner, whereas they decreased with increasing concentrations of DFO in a concentration-dependent manner $(\mathrm{P}<0.05$ for all comparisons; Fig. 2). Western blot analysis demonstrated the same pattern of FPN1 expression at the protein level (Fig. 3). Immunofluorescence analysis revealed that the intensity of FPN1 fluorescence in the cells treated with $50 \mu \mathrm{mol} / 1 \mathrm{FAC}$ was significantly increased when compared with that of the control. In addition, the fluorescence intensity in the cells treated with $10 \mu \mathrm{mol} / \mathrm{l}$ DFO was significantly decreased when compared with that of the control (Fig. 4).

\section{Discussion}

Previous studies have demonstrated that the expression of intracellular FPN1 may be regulated by iron levels in a number of cell types. In 2002, Yang et al (25) found that the mRNA expression of FPN1 was significantly increased in human lung macrophages treated with excessive iron using in situ hybridization. In 2003, Knutson et al (18) reported that iron 
A
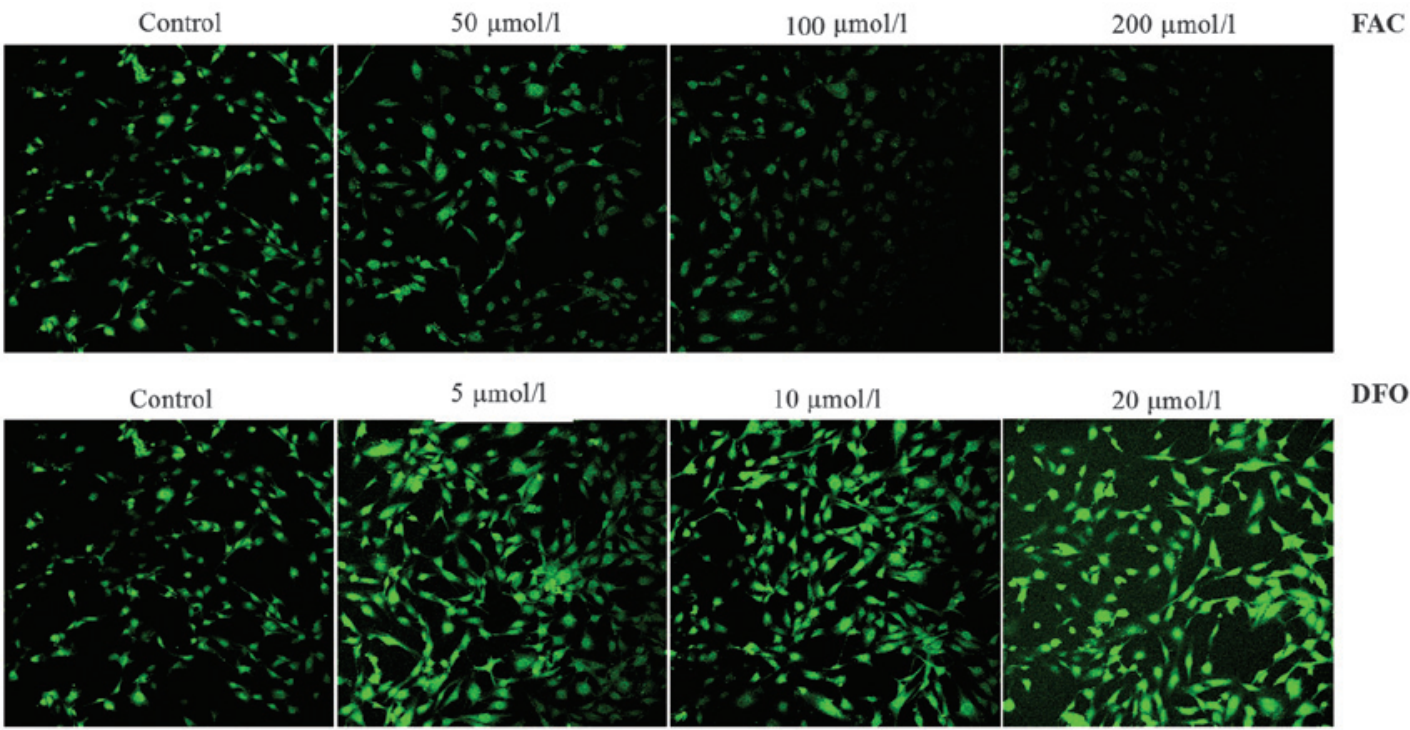

$5 \mu \mathrm{mol} / 1$

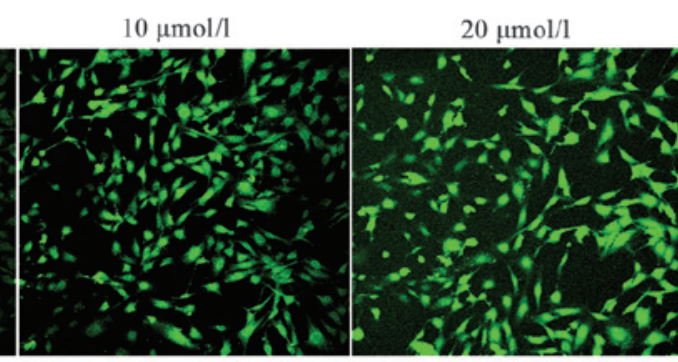

DFO

B
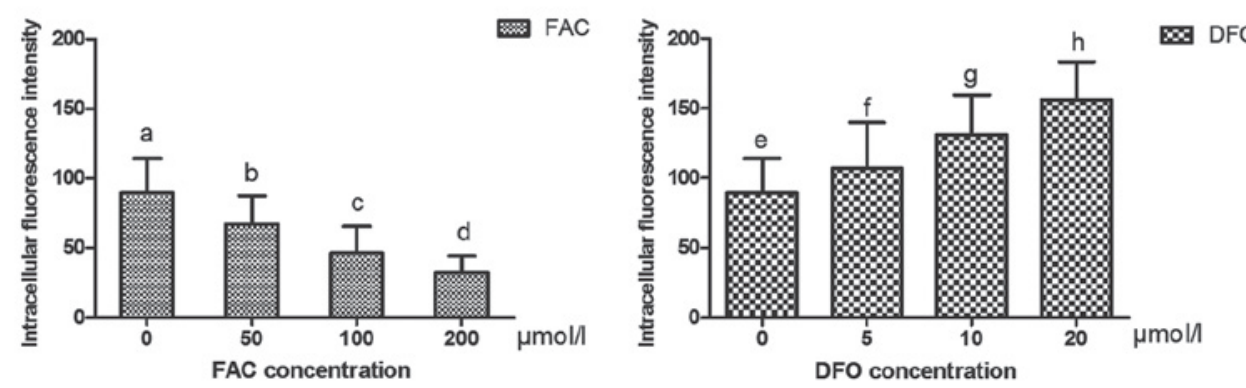

Figure 1. Confocal microscopy analysis of the iron concentration in osteoblasts. (A) The quenching of the fluorescence of Phen Green FL-labeled cells by iron ions was measured with a confocal laser-scanning microscope. Representative microscopic fields are shown (magnification, x20). (B) A correlation between fluorescence intensity and intracellular iron concentration was observed; the fluorescence intensity significantly weakened with increasing FAC concentrations, but was enhanced with increasing of DFO concentrations. Results are expressed as the mean \pm standard deviation of three independent experiments. Means with different letters are significantly different $(\mathrm{P}<0.05)$. FAC, ferric ammonium citrate; DFO, desferrioxamine. a-h indicate that all the values in each bar graph are statistically significantly different.

A

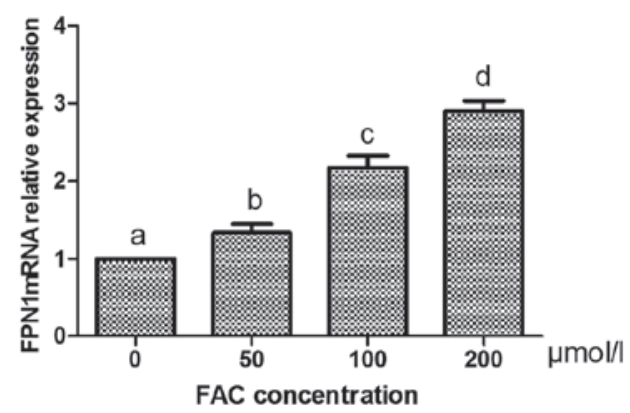

B

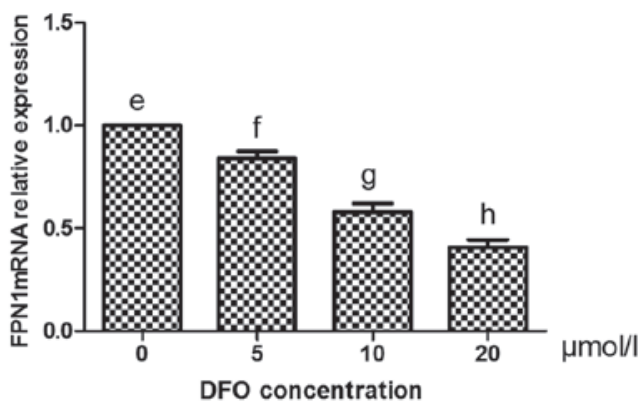

Figure 2. Effect of excessive iron and iron deficiency on the mRNA expression of FPN1 in osteoblasts. (A) RNA expression levels of FPN1 in osteoblasts increased with increasing FAC concentrations in a concentration-dependent manner. (B) mRNA expression levels decreased with increasing DFO concentrations in a concentration-dependent manner. Results are expressed as the mean \pm standard deviation of three independent experiments. Means with different letters are significantly different $(\mathrm{P}<0.05)$. FAC, ferric ammonium citrate; DFO, desferrioxamine; FPN1, ferroportin 1. a-h indicate that all the values in each bar graph are statistically significantly different.

excess increased the mRNA expression levels of FPN1, while iron deficiency decreased the mRNA expression levels of FPN1 in murine J744 macrophages. Furthermore, this effect was completely inhibited by actinomycin D, an inhibitor of RNA polymerase. The results of these studies indicated that the regulation of FPN1 by iron occurs at a transcriptional level in macrophages. In the present study, iron ions were added to the medium in the form of FAC, and a chelator of iron ions was added in the form of DFO. The iron content of the treated cells was measured by CLSM and the results confirmed that iron excess or iron deficiency was achieved in osteoblasts treated with FAC or DFO, respectively. Iron excess was shown to increase the mRNA expression of FPN1 in osteoblasts, while iron deficiency decreased the mRNA expression 
A
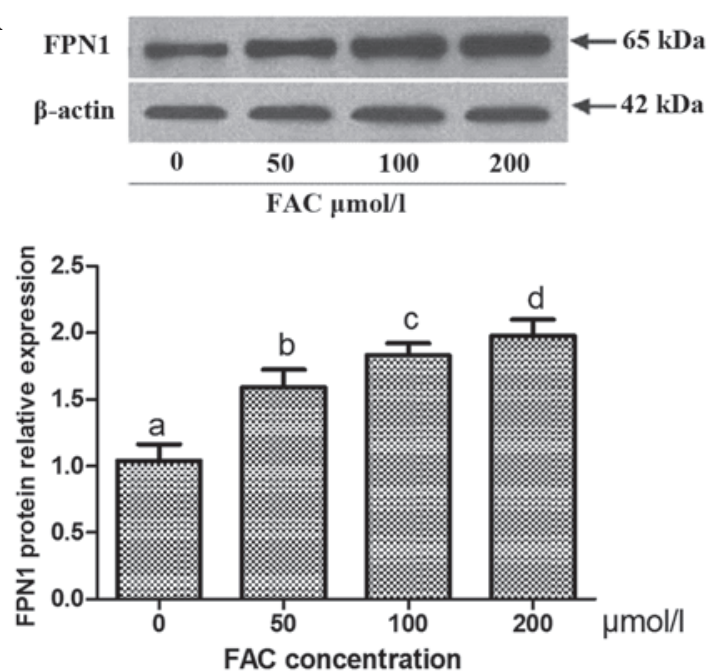
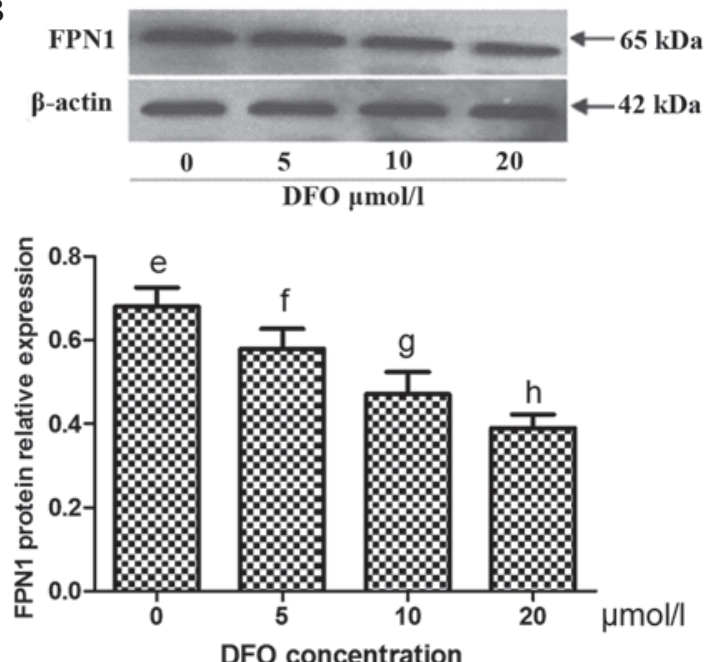

Figure 3. Effect of excessive iron and iron deficiency on the protein expression of FPN1 in osteoblasts. Representative agarose gel images and quantitative analyses showing the protein expression levels of FPN1 in cells treated with (A) FAC and (B) DF. FPN1 protein expression increased with increasing FAC concentrations in a concentration-dependent manner, and decreased with increasing DFO concentrations in a concentration-dependent manner. Results are expressed as the mean \pm standard deviation of three independent experiments. Means with different letters are significantly different (P<0.05). FAC, ferric ammonium citrate; DFO, desferrioxamine; FPN1, ferroportin 1. a-h indicate that all the values in each bar graph are statistically significantly different.

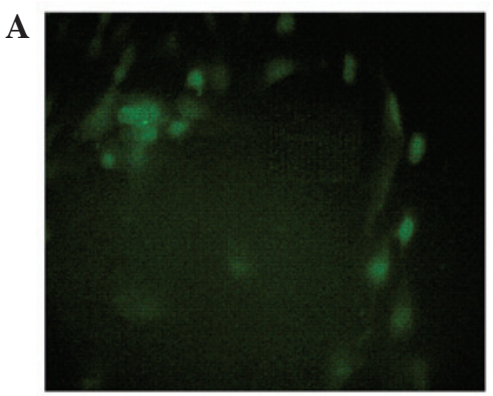

Control

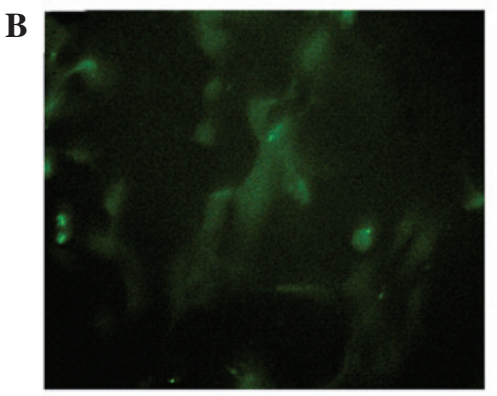

FAC $50 \mu \mathrm{mol} / \mathrm{l}$

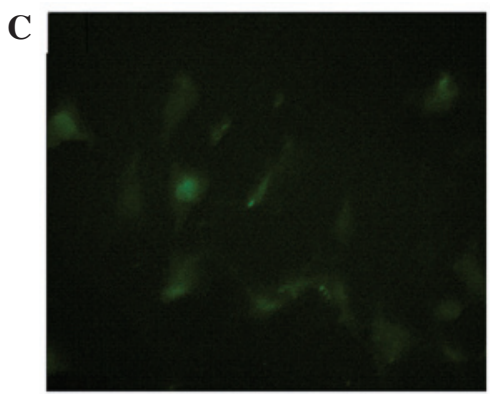

DFO $10 \mu \mathrm{mol} / \mathrm{l}$

Figure 4. Immunofluorescence analysis of FPN1 protein expression in osteoblasts. When compared with the (A) control group, the intensity of FPN1 fluorescence in cells treated with (B) $50 \mu \mathrm{mol} / 1 \mathrm{FAC}$ was significantly increased, while the fluorescence intensity in cells treated with (C) $10 \mu$ mol/1 DFO was significantly decreased (magnification, x40). FAC, ferric ammonium citrate; DFO, desferrioxamine; FPN1, ferroportin 1.

of FPN1 in osteoblasts. These observations indicated that the regulation of FPN1 by iron also occurs at a transcriptional level in osteoblasts. The mechanism underlying regulation at a transcriptional level has been demonstrated to be associated with nuclear factor erythroid-derived 2-like 2 (Nrf2), a transcriptional activator (26). In the case of iron excess, oxidative stress was increased, resulting in Nrf2 nuclear accumulation and the promotion of FPN1 mRNA transcription. In the case of iron deficiency, oxidative stress was decreased, leading to a reduction in Nrf2 expression, thereby the transcription of FPN1 mRNA was inhibited (27).

In recent years, a number of studies have demonstrated that the 5'-untranslated region (UTR) of FPN1 mRNA contains an iron responsive element (IRE). This structure indicates that the regulation of FPN1 expression may occur at a translational level, in a similar manner to ferritin and mitochondrial aconitase $(28,29)$. The presence of the IRE in the 5'-UTR of the mRNA functions as a negative regulator of translation. When intracellular iron levels are high, the activity levels of iron regulatory proteins (IRPs) decrease and, thus, are unable to bind to the 5'-IRE, leading to increased translation of FPN1
mRNA and a release of iron. By contrast, when intracellular iron levels are low, IRPs bind to the 5'-IRE and inhibit the translation of FPN1 mRNA, which results in the decreased release of iron (30). The IRE of FPN1 mRNA has been shown to be functional in a variety of cell types, including the human monocytic cell line U937 (31), and the mouse macrophage cell line RAW264.7 (32). Furthermore, the expression of FPN1 has been shown to be unaffected by iron treatment in HepG2 and Caco- 2 cells with knockout IRE (31). The present study demonstrated that with changes to the intracellular iron content, the expression of PFN1 at the protein level also changes. Therefore, we hypothesized that control of the level of translation may also be involved in the regulation of FPN1 expression in osteoblasts. The regulation of FPN1 at a transcriptional and translational level may be conductive to the balance of intracellular iron. In addition, this process is useful in decreasing iron-mediated oxidative stress to osteoblasts.

In the present study, excessive iron was shown to increase the expression of FPN1, while iron deficiency decreased the expression of FPN1 in osteoblasts. Similar results have also been observed in bronchial epithelial cells (25), macrophages (18-20), 
cardiocytes (24) and hepatocytes (23). However, the expression of FPN1 exhibited opposite effects in enterocytes $(21,22)$ and placental syncytiotrophoblast cells (33), where excessive iron decreased the expression of FPN1 and iron deficiency increased the expression of FPN1. This difference may be associated with cell type. For example, intestinal epithelial cells and placental cells are responsible for the transportation of exogenous iron into plasma; thus, the molecular mechanisms underlying iron metabolism may be different from those of other cells. In addition, Marro et al (34) reported that FAC added in vitro did not affect the expression of FPN1 in the mouse macrophage cell line RAW 264.7. However, the results of the present study are not consistent with these observations, which may be explained by differences in the concentrations of FAC. In the previous study, $2 \mu \mathrm{mol} / \mathrm{F} \mathrm{FAC}$ was applied; this concentration was $10-100$ fold lower than that applied in the present study. The normal serum iron concentration in the human body is $12.5-30 \mu \mathrm{mol} / 1$, and in the case of an iron overload, the concentration can increase to $>50 \mu \mathrm{mol} / 1$. Therefore, FAC at a higher concentration may simulate iron overload in vivo more closely.

In conclusion, excessive iron increased the expression of FPN1 in osteoblasts, while iron deficiency decreased the expression of FPN1 in osteoblasts. The regulation of FPN1 in osteoblasts by iron may occur at transcriptional and translational levels. These observations indicate that FPN1 plays an important role in iron metabolism in osteoblasts.

\section{Acknowledgements}

The study was partially supported by grants from the National Natural Science Foundation of China (no. 81273090), Jiangsu Provincial Grant (no. BK2012608), the Social Development Fund of Jiangsu Province (no. BE2011605) and the Research and Innovation Project for College Graduates of Jiangsu Province (no. CXZZ12_0845).

\section{References}

1. Weinberg ED: Iron loading: a risk factor for osteoporosis. Biometals 19: 633-635, 2006.

2. Weinberg ED: Role of iron in osteoporosis. Pediatr Endocrinol Rev 6 (Suppl 1): 81-85, 2008.

3. Jian J, Pelle E and Huang X: Iron and menopause: does increased iron affect the health of postmenopausal women? Antioxid Redox Signal 11: 2939-2943, 2009.

4. Liu G, Men P, Kenner GH and Miller SC: Age-associated iron accumulation in bone: implications for postmenopausal osteoporosis and a new target for prevention and treatment by chelation. Biometals 19: 245-251, 2006.

5. Liu G, Men P, Kenner GH and Miller SC: Therapeutic effects of an oral chelator targeting skeletal tissue damage in experimental postmenopausal osteoporosis in rats. Hemoglobin 32: 181-190, 2008.

6. Li GF, Pan YZ, Sirois P, et al: Iron homeostasis in osteoporosis and its clinical implications. Osteoporos Int 23: 2403-2408, 2012.

7. Doyard M, Fatih N, Monnier A, et al: Iron excess limits HHIPL-2 gene expression and decreases osteoblastic activity in human MG-63 cells. Osteoporos Int 23: 2435-2445, 2012.

8. Messer JG, Kilbarger AK, Erikson KM and Kipp DE: Iron overload alters iron-regulatory genes and proteins, down-regulates osteoblastic phenotype, and is associated with apoptosis in fetal rat calvaria cultures. Bone 45: 972-979, 2009.

9. Yamasaki K and Hagiwara H: Excess iron inhibits osteoblast metabolism. Toxicol Lett 191: 211-215, 2009.

10. Messer JG, Cooney PT and Kipp DE: Iron chelator deferoxamine alters iron-regulatory genes and proteins and suppresses osteoblas phenotype in fetal rat calvaria cells. Bone 46: 1408-1415, 2010 .
11. Pattanapanyasat K, Webster HK, Tongtawe P, et al: Effect of orally active hydroxypyridinone iron chelators on human lymphocyte function. Br J Haematol 82: 13-19, 1992.

12. Donovan A, Lima C and Andrews NC: Analysis of iron homeostasis in mice with a targeted deletion of the gene encoding the iron exporter Ferroportin 1. Blood 102: 157a, 2003.

13. Ganz T: Cellular iron: ferroportin is the only way out. Cell Metab 1: 155-157, 2005.

14. Rice AE, Mendez MJ, Hokanson CA, et al: Investigation of the biophysical and cell biological properties of ferroportin, a multipass integral membrane protein iron exporter. J Mol Biol 386: 717-732, 2009.

15. Canonne-Hergaux F, Donovan A, Delaby C, et al: Comparative studies of duodenal and macrophage ferroportin proteins. Am J Physiol Gastrointest Liver Physiol 290: G156-G163, 2006.

16. Yang F, Liu XB, Quinones M, et al: Regulation of reticuloendothelial iron transporter MTP1 (Slc11a3) by inflammation. J Biol Chem 277: 39786-39791, 2002.

17. Xu Y, Zhang W, Zhang P, et al: Downregulation of ferroportin 1 expression in hFOB 1.19 osteoblasts by hepcidin. Inflammation 35: 1058-1061, 2012.

18. Knutson MD, Vafa MR, Haile DJ and Wessling-Resnick M: Iron loading and erythrophagocytosis increase ferroportin 1 (FPN1) expression in J774 macrophages. Blood 102: 4191-4197, 2003.

19. Delaby C, Pilard N, Hetet G, et al: A physiological model to study iron recycling in macrophages. Exp Cell Res 310: 43-53, 2005.

20. Delaby C, Pilard N, Gonçalves AS, et al: Presence of the iron exporter ferroportin at the plasma membrane of macrophages is enhanced by iron loading and down-regulated by hepcidin. Blood 106: 3979-3984, 2005.

21. Thomas C and Oates PS: Ferroportin/IREG-1/MTP-1/SLC40A1 modulates the uptake of iron at the apical membrane of enterocytes. Gut 53: 44-49, 2004.

22. Zoller H, Theurl I, Koch R, et al: Mechanisms of iron mediated regulation of the duodenal iron transporters divalent metal transporter 1 and ferroportin 1. Blood Cells Mol Dis 29: 488-497, 2002.

23. Balusikova K, Neubauerova J, Dostalikova-Cimburova M, et al: Differing expression of genes involved in non-transferrin iron transport across plasma membrane in various cell types under iron deficiency and excess. Mol Cell Biochem 321: 123-133, 2009.

24. Qian ZM, Chang YZ, Leung G, et al: Expression of ferroportin1, hephaestin and ceruloplasmin in rat heart. Biochim Biophys Acta 1772: 527-532, 2007.

25. Yang F, Wang X, Haile DJ, et al: Iron increases expression of iron-export protein MTP1 in lung cells. Am J Physiol Lung Cell Mol Physiol 283: L932-L939, 2002.

26. Nairz M, Schleicher U, Schroll A, et al. Nitric oxide-mediated regulation of ferroportin-1 controls macrophage iron homeostasis and immune function in Salmonella infection. J Exp Med 210: $855-873,2013$

27. Harada N, Kanayama M, Maruyama A, et al: Nrf2 regulates ferroportin 1-mediated iron efflux and counteracts lipopolysaccharide-induced ferroportin 1 mRNA suppression in macrophages. Arch Biochem Biophys 508: 101-109, 2011

28. McKie AT, Marciani P, Rolfs A, et al: A novel duodenal iron-regulated transporter, IREG1, implicated in the basolateral transfer of iron to the circulation. Mol Cell 5: 299-309, 2000.

29. Donovan A, Brownlie A, Zhou Y, et al: Positional cloning of zebrafish ferroportin 1 identifies a conserved vertebrate iron exporter. Nature 403: 776-781, 2000.

30. Eisenstein RS: Iron regulatory proteins and the molecular control of mammalian iron metabolism. Annu Rev Nutr 20: 627-662, 2000.

31. Lymboussaki A, Pignatti E, Montosi G, et al: The role of the iron responsive element in the control of ferroportin1/IREG1/MTP1 gene expression. J Hepatol 39: 710-715, 2003.

32. Liu XB,Hill Pand Haile DJ: Role of the ferroportin iron-responsive element in iron and nitric oxide dependent gene regulation. Blood Cells Mol Dis 29: 315-326, 2002.

33. Li YQ, Bai B, Cao XX, et al: Ferroportin 1 and hephaestin expression in BeWo cell line with different iron treatment. Cell Biochem Funct 30: 249-255, 2012.

34. Marro S, Chiabrando D, Messana E, et al: Heme controls ferroportin1 (FPN1) transcription involving Bach1, Nrf2 and a MARE/ARE sequence motif at position -7007 of the FPN1 promoter. Haematologica 95: 1261-1268, 2010. 